\title{
An Indoor IoT based Location Aware System
}

\author{
Prof. B.B. Gite ${ }^{1}$, Swati Pandey ${ }^{2}$ \\ Head of Dept., Computer Dept., Sinhgad Academy of Engg, Kondhawa, Pune, India ${ }^{1}$ \\ Student, Computer Dept., Sinhgad Academy of Engg, Kondhawa, Pune, India ${ }^{2}$
}

\begin{abstract}
The new technologies characterizing the internet of Things permit realizing real sensible environments ready to offer advanced services to the users. Recently, these sensible environments also are being exploited to renovate the users' interest on the cultural heritage, by guaranteeing real interactive cultural experiences. During this paper, we have a tendency to style and validate an inside location-aware design ready to enhance the user expertise during adiposity. Above all, the projected system depends on a wearable device that mixes image recognition and Localization capabilities to mechanically offer the users with cultural contents associated with the determined artworks. The localization data is obtained by a Bluetooth infrastructure put in within the Museum. Moreover, the system interacts with the Cloud to store multimedia system contents created by the user and to share environment-generated events on his/her social networks.
\end{abstract}

Keywords: Bluetooth, Image recognition, IoT, Tracking system, Location-awareness, SoA, Smart Museum.

\section{INTRODUCTION}

The purpose of the museum project was to enhance access to the scientific collections and to upgrade internal info by making joint national databases. Ideally, the information systems would manage all reference info referring to the museums internal and external collections. During this means, the museums' internal necessities for assortment management, research, field work, education and communication would be coated. It had been additionally requested that the systems ought to suits the external demands of the authorities and society relating to open access to info concerning our common cultural and natural heritage. The bulk of the reference info within the museums existed on paper.

\section{LITERATURE SURVEY}

A. A location aware personalized smart control system Smart management systems infer user locations through wireless detector networks, active and passive RFIDs, NFCs etc., to call some. This involves considerable quantity of installation and detector information managing. We have a tendency to gift a personalised sensible system that learns user location and controls appliances gift at the user's location. The sole detector we have a tendency to use is that the smart phone itself through its embedded field of force and light-weight sensors for classifying user locations and sleuthing intensity level in rooms severally.

B. A location-aware lifestyle improvement system to save energy in smart home

Smart-home may be a terribly helpful application in IoT to manage electronic devices and electrical appliances uniformly in residence. Even there square measure some approaches to avoid wasting energy in sensible homes, there's an absence of effective solutions for saving energy by ever-changing users' habit. Thus we have a tendency to propose a system for up the approach to life habit to avoid wasting the energy during this paper. This technique lets users United Nations agency board the smart-home bear in mind of their life vogue, wasted electricity, so improves their modus Vivendi. The system initial detects varied data, e.g., position of the user, and energy-usage of home electrical appliances. And then, the system acknowledges state of affairs of wasting energy and supply services to enhance the approach to life of users.

C. An IoT-based User-centric Ecosystem for Heterogeneous Smart Home Environments.

Recent innovations within the ICT field square measure powerfully targeted towards the net of Things, which is able to undoubtedly cause Associate in nursing sweetening additionally within the domestic environments. Lowpower and cheap devices square measure expected to form a network of interconnected sensible objects ready to remodel our homes into real sensible Homes. However, the no uniformity of the underlying technologies prevents these sensible objects to natively interoperate for adapting the atmosphere to users' wants. additionally, common users square measure typically excluded from the event of latest applicable services that exploit physical devices, as they are doing not have sufficient programming and technological skills

\section{III.SYSTEM ARCHITECTURE}

Over the centuries, many museums and art galleries have preserved our various cultural heritages and served as necessary sources of education and learning. Particularly, it's tough to outline earlier a tour for all the visitors, as a result of interests could vary from person to person. Therefore, interactive and personalised repository tours ought to be developed. Finally, many location-aware 
Vol. 5, Issue 12, December 2016

services, running within the system, management the atmosphere standing additionally in line with users' movements. These services act with physical devices through a multi-protocol middleware. The system has been designed to be simply extensile to alternative IoT technologies and its effectiveness. The effectiveness of the planned design is evaluated in 2 sequential phases. First, the performance of each the image recognition rule and therefore the localization service is analysed through specific stressing tests, whereas the full design is evaluated in an exceedingly real situation staged at museum.

\section{IV.SYSTEM ANALYSIS}

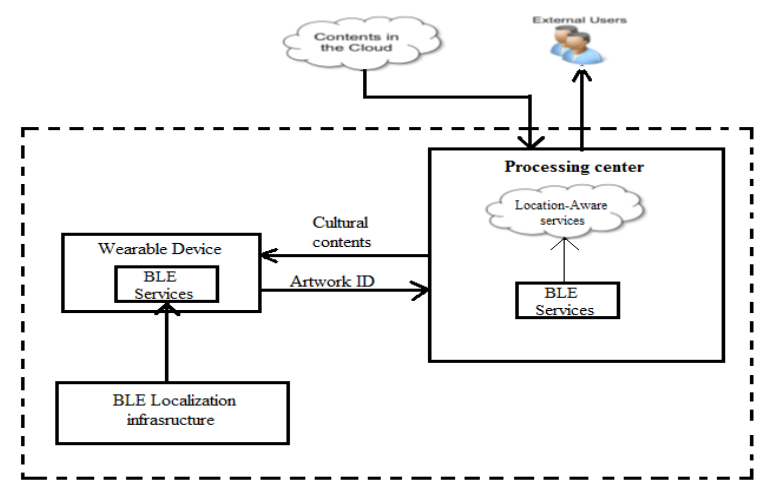

Fig. 1 shows the overall structure of the proposed system architecture. It is composed, as described below, of two main building blocks:

\section{- The localization service:}

Here, the localization info is keep and created accessible to different services.

\section{- The processing centre:}

It is the core of the business logic. It accesses, within the Cloud, the cultural contents needed by the users and well provides such contents on many interactive platforms. Then, it permits the execution of many location-aware services by providing them with the positioning data returning from the localization infrastructure.

\section{- Indoor localization :}

As said, many system parts depend upon the localization service. It consists of 3 main elements: (i) Associate in infrastructure of wireless landmarks that sporadically send localization info, (ii) a service put in on the wearable good entrance that collects the data of the landmarks to see its location, and (iii) the service running on the process center that receives the situation of the user and provides it to the opposite services. Mores pacifically, the network of wireless landmarks consists of embedded devices equipped with Bluetooth Low Energy(BLE) interface and placed separately within the completely different rooms of the building. The selection of BLE is principally attributable to its low energy consumption before of a communication vary comparable with that of the standard Bluetooth. Each device of the BLE infrastructure sends its location identification (ID) along with the transmission
(TX) power value. The service running on the user's wearable device collects location knowledge from all the landmarks at intervals its listening vary and so determines the area during which it's located. The interpretation of the design symbol will result in 2 potential results:

1. Associate audio description of the design on the user's wearable device;

2. Multimedia cultural contents on interactive walls of the museum

\section{CONCLUSION}

In this work, an interior location-aware design for smart museums was designed and valid. In additional detail, the projected system depends on a wearable device equipped with image recognition and localization capabilities to mechanically offer users with cultural contents associated with the determined artworks. The power to spot the user's position is secure by associate degree infrastructure of Bluetooth Low Energy transmitters. The design additionally consists of a process centre, wherever the particular business logic is responsible to (i) retrieve from the Cloud the cultural info associated with the determined artworks and (ii) manage the standing of the indoor surroundings in accordance to users' position.

\section{ACKNOWLEDGEMENT}

I would like to thank all the researchers working on this field who in one way or another guided us on achieving our goals. We would also like to thank all the professors at Sinhgad Academy of Engineering (Savitribai Phule Pune University, Pune) who were kind enough to share their views, some suggestions in making this project success.

\section{REFERENCES}

[1] M.V.Moreno, J.L.Hernandez, and A.F.Skarmeta, "A New LocationAware Authorization Mechanism for Indoor Environments," in Proc. 28th International Conference on Advanced Information Networking and Applications, Victoria, 2014, pp. $791-796$.

[2] L.Mainetti, V.Mighali, and L. Patrono, "An IoT-based User-centric Ecosystem for Heterogeneous Smart Home Environments," in Proc. 2015 IEEE International Conference on Communications, IEEE ICC 2015, London (UK), June 8-12, 2015.

[3] L. Mainetti, V. Mighali, L. Patrono, "A Location-aware Architecture for Heterogeneous Building Automation Systems, “ in 14th IFIP/IEEE Symposium on Integrated Network and Service Management, IM 2015, Ottawa (Canada), May 11-15, 2015.

[4] G. De Luca, P. Lillo, L. Mainetti, V. Mighali, L. Patrono, and I. Sergi, "The use of NFC and Android technologies to enable a KNX-based smart home," in Proc. 2013 International Conference on Software, Telecommunications and Computer Networks, SoftCOM 2013, 2013, Article number 6671904, pp.1-7.

[5] M. Jung, J. Weidinger, C. Reinisch, W. Kastner, C. Crettaz, A. Olivieri, and Y. Bocchi, "A Transparent IPv6 Multi-protocol Gateway to Integrate Building Automation Systems in the Internet of Things," in Proc. 2012 IEEE Int. Conf. Green Computing and Communications,

[6] Besancon, 2012, pp. 225 - 233.

[7] M. Jung, C. Reinisch, and W. Kastner, "Integrating Building Automation Systems and IPv6 in the Internet of Things," in Proc. Sixth Int. Conf. Inn. Mobile and Internet Services in Ubiquitous Computing, Palermo, 2012, pp. $683-688$. 